\title{
Compensator Control For Chemical Vapor Deposition Film Growth Using Reduced Order Design Models
}

\author{
G.M. Kepler, H.T. Tran, H.T. Banks* \\ Center For Research in Scientific Computation \\ North Carolina State University, Raleigh, NC ${ }^{27695}$
}

\begin{abstract}
We present a summary of investigations on the use of proper orthogonal decomposition (POD) techniques as a reduced basis method for computation of feedback controls and compensators in a high pressure chemical vapor deposition (HPCVD) reactor that includes multiple species and controls, gas phase reactions, and time dependent tracking signals that are consistent with pulsed vapor reactant inputs. Numerical implementation of the model-based feedback control uses a reduced order state estimator, based on partial state observations of the fluxes of reactants at the substrate center, which can be achieved with current sensing technology. We demonstrate that the reduced order state estimator or compensator system is capable of substantial control authority when applied to the full system.

* Corresponding author: H.T. Banks, Center For Research in Scientific Computation, Box 8205, NCSU, Raleigh NC 27695

Telephone, 919-515-3968; Fax, 919-515-1636; Email, htbanks@eos.ncsu.edu
\end{abstract}




\section{Introduction}

Stringent control of layer thickness and composition is essential in the production of advanced optoelectronic integrated circuits. This can sometimes be addressed, in part, through open-loop optimization [7, 25, 41]. However, process variability and the increasing demands put on control of layer thickness and composition in state-of-the art devices increase the desirability of real-time control of film growth [8, 19, 21, 43, 42]. Low pressure chemical vapor deposition (CVD) processes are the preferred choice for manufacturing many advanced optoelectronic devices. On the other hand, there are also materials of potential industrial use (e.g., InN films) that exhibit relatively high decomposition pressure as compared to other III-V compounds. These can not be adequately produced at desirable process temperatures under low pressure conditions and thus it is be desirable to extend the CVD processing to higher pressures. We are presently collaborating with material scientists at N.C. State University to design and build such a HPCVD reactor with real-time sensing and control as an innovative feature of this prototype reactor. Previous work within this collaboration has experimentally demonstrated the successful implementation of closed-loop control of film thickness and composition in $\mathrm{GaP} / \mathrm{Ga}_{1-x} \operatorname{In}_{x} \mathrm{P}$ heterostructures grown in a low pressure pulsed chemical beam epitaxy $(\mathrm{CBE})$ reactor utilizing real-time optical p-polarized reflectance signal (PRS) sensing [19].

The modeling of reactant transport in HPCVD reactors is more complicated than in the case of low pressure CVD reactors. In general, a full mathematical model describing transport processes in (HPCVD) systems is given by a system of nonlinear partial differential equations representing the continuity, momentum, energy, and species equations of state. Numerical simulations and control designs of such systems using finite element, finite difference, or spectral methods lead to very large systems of ordinary differential equations rendering real-time full model-based feedback control design infeasible. Substantial dimensional reduction can be realized using the method of POD to more efficiently represent the system data [33]. In recent work, we demonstrated the potential of a POD based reduced order model as a basis for implementing real time control by presenting a method for the design of state feedback controllers based on data provided by accurate model simulations of species transport [26].

In this paper we extend the design of state feedback controllers to include experimentally relevant conditions of high pressure III-V film growth with multiple species and controls, gas phase reactions, and time dependent tracking signals that are consistent with pulsed vapor reactant inputs. We present implementation of Dirichlet boundary control of dilute Group III and Group V reactants transported by convection and diffusion to an absorbing substrate while undergoing gas phase reactions. Computational fluid dynamics (CFD) simulations provide data on the full system and are used to construct a POD reduced 
order model.

The control/compensator problem (or LQG tracking problem) is formulated as a linear quadratic regulator (LQR) time dependent tracking problem. The state of the system is estimated via a compensator gain from observations of the fluxes of Group III and Group $\mathrm{V}$ reactants to the substrate, which are assumed to be proportional to the growth rate, with the observed fluxes attempting to track time dependent target flux values such as is encountered in pulsed CVD film growth. For example, one might choose a target flux such that the integrated pulse deposits enough material to form a monolayer. Dirichlet boundary control at the inlet determines the mass fractions of the incoming Group III and Group V reactants. We demonstrate that the reduced order state estimator or compensator system is capable of substantial control authority when applied to the full system.

The use of POD (also known under the names of principal component analysis [23] and Karhunen-Loève expansion $[24,30]$ ) as a method of feature extraction from data sets is well known in statistical and pattern recognition fields [20] and has been applied in a wide variety of areas, such as materials processing [33, 41], characterization of human faces [37], and turbulent coherent flows $([4,15,16,18,22,31,38]$ - see also the surveys $[14,32])$. The POD method is a linear transformation of a multivariate data set into an optimal set of uncorrelated variables (POD modes). The original multivariate data can be written as linear combinations of the POD modes. In many cases the POD modes more efficiently describe the variability of the original data and some dimensional reduction is possible by retaining only the most important modes.

Recent use of POD for reduction of order in distributed parameter systems includes applications to parameter estimation or inverse problems [11] and both open loop and feedback control design $[1,2,9,10,26,27,33,34,41]$. There are a number of nontrivial issues related to the use of reduced basis methods in general, and POD methods in particular, as a foundation for approximation methods in infinite dimensional systems such as those modeled by distributed parameter or partial differential equation systems. The most important issue is whether the infinite dimensional system can be approximated well by a finite span of basis elements. Initial efforts on error analysis for POD based approximations in forward or simulation problems can be found in [28]. A more fundamental question in regard to order reduction is whether the important features of the system are essentially low finite dimensional in nature? There is mounting computational evidence that the answer to this question is positive for many structural systems and for a substantial number of fluid and electromagnetic applications [11]. However, even when this question can be answered in the affirmative, it is not at all clear that one can use such an approach for control design. 
Specifically, if one uses 'snapshots' of the uncontrolled system (as was done in $[9,10]$ ) to construct the POD basis elements, there is little reason to expect that control design based on this finite dimensional approximation will be effective when applied to the original system. The controlled original system itself may require a different set of finite dimensional elements for efficient approximation or, worse yet, may not be amenable to a low order basis approximation. Fortunately, the results of $[9,10]$ suggest that, at least in some situations, this is not the case. Another approach is to 'snapshot' on the system under several levels of nontrivial control inputs that are not, in general, derived from any optimal or suboptimal design. This approach has proved effective in open loop [33] as well as closed loop [26] control problems and is employed in this paper.

In Section 2 below we describe the particular system under investigation here and describe the simulations needed to develop POD based control design. A sample of some of our computational results based on this approach is given in Section 3. Brief conclusions are then summarized.

\section{Methods}

We consider a 2D rectangular domain (Fig. 1) representing the longitudinal cross section through the center of an HPCVD reactor with the following dimensions: $0.011 \mathrm{~m}$ height, $0.156 \mathrm{~m}$ total length, and $0.048 \mathrm{~m}$ substrate length. Gas flow enters from the inlet on the left of the domain $\left(\Gamma_{1}\right)$ and exits at the right boundary of the domain $\left(\Gamma_{3}\right)$, after passing over the heated substrate on the bottom wall $\left(\Gamma_{5}\right)$. We control the concentration of the source material at the inlet in an effort to obtain a desired flux of reactants to the substrate, which can be assumed to be proportional to the growth rate. The remainder of

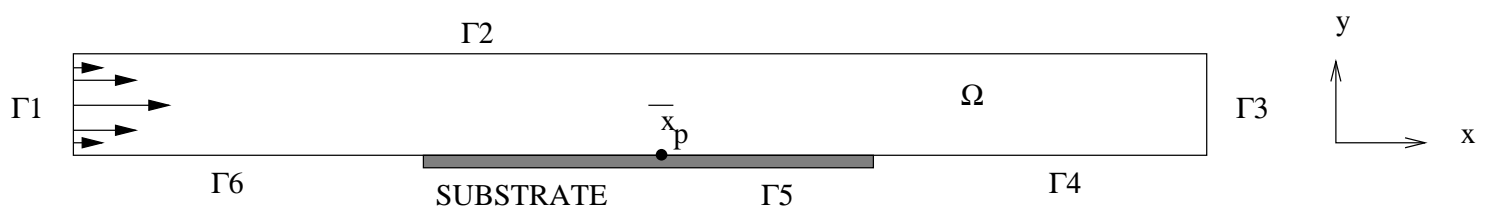

Figure 1: Two-dimensional cross section of HPCVD reactor geometry with the following dimensions height $=0.011 \mathrm{~m}$, length $=0.156 \mathrm{~m}$, substrate length $=0.048 \mathrm{~m}$.

the Methods section is organized into two parts. The first presents methods for obtaining simulated experimental data or time snapshots necessary for obtaining the POD modes for the reduced order model, while the second section presents the control equations. 


\subsection{Simulated data}

We consider only trace amounts of reactants mixed with the carrier gas. Under this dilute approximation, we can obtain steady-state solutions for the velocity, temperature, and pressure that are independent of the reactant concentration and are governed by the following set of equations (continuity)

$$
\vec{\nabla} \cdot(\rho \vec{v})=0
$$

(momentum)

$$
\rho \vec{v} \cdot \vec{\nabla} \vec{v}=-\vec{\nabla} P+\vec{\nabla} \cdot \vec{\tau}-\rho \vec{g},
$$

where the viscous stress tensor is of the form

$$
\vec{\tau}=-\frac{2}{3} \mu(\vec{\nabla} \cdot \vec{v}) \vec{I}+\mu\left(\vec{\nabla} \vec{v}+\vec{\nabla} \vec{v}^{T}\right)
$$

(energy)

$$
\rho c_{p} \vec{v} \cdot \vec{\nabla} T=\vec{\nabla} \cdot(k \vec{\nabla} T),
$$

where $\vec{g}$ is the gravitational acceleration, $\vec{u}, T$, and $P$ are the velocity, temperature, and pressure, $\mu, c_{p}$, and $k$ are the viscosity, specific heat, and thermal conductivity of the carrier gas. The density variations are modeled as $\rho=\rho_{0}\left[1-\beta\left(T-T_{0}\right)\right]$, where $T_{0}$ is a reference temperature, $\rho_{0}$ is a reference density calculated from the ideal gas law at the reference temperature and reactor pressure, and $\beta$ is the volume coefficient of expansion $(\beta=1 / T)$. The boundary conditions for the above system of equations (1)-(4) will be discussed in subsequent sections.

The governing equations are discretized using the Galerkin finite element method with weighted residuals for the degrees of freedom $(\vec{v}, P$, and $T)$. A mixed formulation with 132 quadrilateral elements (corresponding to 453 nodes) is used with piecewise linear discontinuous elements for pressure, and quadratic (8-noded) elements for the other degrees of freedom. Solutions for the variables $\vec{v}, P, T$, and $Y_{n}$ are obtained using simulations with commercially available code (FIDAP, Fluid Dynamics International, Evanston, IL) on a Silicon Graphics Power Indigo 2.

For the results presented in this article, we consider a hydrogen carrier gas at atmospheric pressure. Temperature dependent values for $\mu, k$, and $c_{p}$ are linearly interpolated from measurements taken from the available literature [29, 39, 13]. A parabolic velocity flow profile is specified at the inlet $(\Gamma 1)$, with an average inlet velocity of $0.1147 \mathrm{~m} / \mathrm{s}$. No slip (zero velocity) boundary conditions are imposed on those portions of the model corresponding to the reactor walls $(\Gamma 2, \Gamma 4, \Gamma 5$, and $\Gamma 6)$. Room temperature boundary conditions are imposed at the inlet and along the upper wall ( $Г 2)$. Along the bottom wall, the substrate $(\Gamma 5)$ temperature is fixed at $800^{\circ} \mathrm{K}$, with a non-linear temperature 
decrease from the substrate edge to the inlet $(\Gamma 6)$ and, similarly, from the substrate edge to the outlet ( $\Gamma 4)$ (see Fig. 1).

Steady-state solutions for $v, T$, and $\rho$ obtained above are used in the time-dependent governing equation for species transport

$$
\frac{\partial Y_{n}}{\partial t}+\vec{v} \cdot \vec{\nabla} Y_{n}=\frac{1}{\rho} \vec{\nabla} \cdot\left(\rho D_{n} \vec{\nabla} Y_{n}\right)+\sum_{i=1}^{N_{R}} r_{n i},
$$

where $D_{n}$ is the diffusivity of the species, $Y_{n}$ is the mass fraction of the $n$th species, $N_{R}$ is the number of gas phase reactions, and $r_{n i}$ is the rate of production of species $n$ in the $i$ th chemical reaction. Solutions of this equation (with boundary conditions to be specified) are used to generate data for construction of the POD modes.

Trimethylindium (TMI) and phosphine are source materials commonly used for CVD growth of III-V films where indium and phosphorus are the constituents. For high pressure applications, the source gases are introduced in separate pulses with sufficient time between pulses to allow the reactants to exit before introduction of the next source material (Fig. 2). Pulsing of the III-V source materials prevents nucleation of the film in the gas phase and makes PRS observation and analysis possible. A constant total volumetric flow rate is maintained throughout the pulsing cycle.

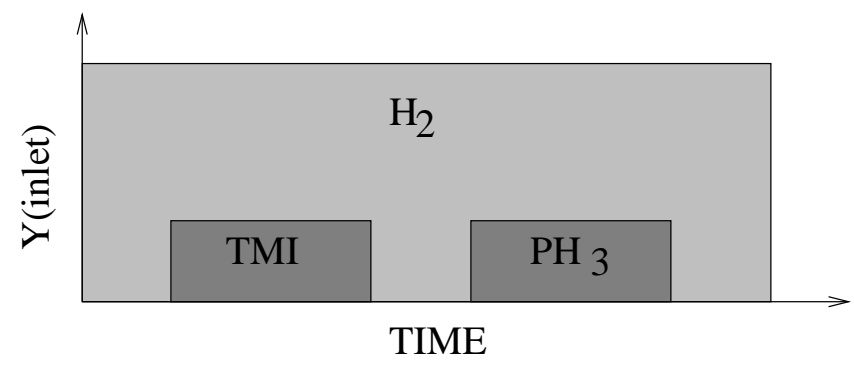

Figure 2: Schematic representation of source gas pulsing.

Under the reactor conditions considered here $\left(\mathrm{H}_{2}\right.$ carrier gas, $800^{\circ} \mathrm{K}$ substrate temperature, and 1 atm pressure), there are no effective gas phase reaction mechanisms for phosphine, and the only significant gas phase reaction for TMI is the decomposition of TMI to monomethylindium (MMI) and two methyl molecules, $\mathrm{In}\left(\mathrm{CH}_{3}\right)_{3} \rightarrow \mathrm{InCH}_{3}+$ $2 \mathrm{CH}_{3}$. This reaction can be described as a first-order Arrhenius reaction

$$
r_{n}=\nu_{n} \frac{W_{n}}{W_{T M I}} k_{0} e^{(-E / R T)} Y_{T M I}
$$

where $\nu_{n}$ refers to the stoichiometry of species $n$ in the reaction, $W_{n}$ and $W_{T M I}$ refer to the molecular weight of species $n$ and TMI, respectively, $k_{0}=5.25 \times 10^{15} \mathrm{~s}^{-1}$ is the rate constant, and $E=47.2 \mathrm{kcal} / \mathrm{mol}$ is the activation energy [40]. 
A transient simulation of TMI transport (5) through one cycle of pulsing and clearing including the thermal decomposition of TMI, diffusion of TMI and MMI, and absorption at the substrate - is used to obtain data for construction of the POD modes for TMI and MMI. Similarly, simulation data of phosphine transport from one pulsing and clearing cycle is used to construct the POD modes for phosphine. The simulation system, including a nonzero boundary input, is given by

$$
\begin{aligned}
\frac{\partial Y_{n}}{\partial t}+\vec{v} \cdot \vec{\nabla} Y_{n} & =\frac{1}{\rho} \vec{\nabla} \cdot\left(\rho D_{n} \vec{\nabla} Y_{n}\right)+\lambda_{n}(T) Y_{1} \\
Y_{n}(0, \vec{x}) & =0 \\
Y_{1}(t, \vec{x}), Y_{2}(t, \vec{x}) & =1 \text { or } 0 \text { (pulsed) on } \Gamma 1 \\
Y_{3}(t, \vec{x}) & =0 \text { on } \Gamma 1 \\
Y_{n}(t, \vec{x}) & =0 \text { on } \Gamma 5 \\
\frac{\partial Y_{n}(t, \vec{x})}{\partial n} & =0 \text { on } \Gamma 2 \cup \Gamma 3 \cup \Gamma 4 \cup \Gamma 6 \\
n & =1,2,3
\end{aligned}
$$

where $Y_{1}, Y_{2}$, and $Y_{3}$ refer to the mass fractions of TMI, phosphine, and MMI, respectively, $\lambda_{1}(T)=-k_{0} e^{(-E / R T)}, \lambda_{2}(T)=0, \lambda_{3}(T)=\left(W_{3} / W_{1}\right) k_{0} e^{(-E / R T)}$, and $\frac{\partial}{\partial n}$ denotes the outward normal derivative. Since the methyl $\left(\mathrm{CH}_{3}\right)$ molecules do not participate in film growth or otherwise affect the transport properties (under the dilute approximation), we do not include them in the transport equations. The reactor walls $(\Gamma 2, \Gamma 4$, and $\Gamma 6)$ are assumed non-absorbing, and the substrate $(\Gamma 5)$ is assumed to be perfectly absorbing (concentration of zero). Values for $\vec{v}$ and $\rho$ appearing in (5) are provided by the steadystate solutions. Temperature dependent values for the diffusivities $D_{n}$ in hydrogen are linearly interpolated from values taken from the available literature [40].

Initially, the reactant mass fractions are assumed zero everywhere in the reactor and only the $\mathrm{H}_{2}$ carrier gas is flowing. At the start of the pulsing cycle, a normalized TMI mass fraction of one $\left(Y_{1}=1\right)$ is maintained as a nontrivial input 'control' at the inlet $(\Gamma 1)$ for two seconds, followed by a clearing pulse of one second with no input 'control' at the inlet (Fig. 2). At the end of the clearing pulse a normalized phosphine mass fraction of one $\left(Y_{2}=1\right)$ is maintained as a second nontrivial input 'control' at the inlet $(\Gamma 1)$ for two seconds, followed by a clearing pulse of one second with no input 'control' at the inlet. Time integration is implemented using a backward Euler method with fixed time steps $(0.02 \mathrm{~s})$. Intermediate solution values are stored at each time step to be used later in construction of the POD basis elements.

Construction of POD Modes The reactant transport simulation described above provides a multivariate data set consisting of $K$ vectors $X_{n}=\left\{x_{n 1}^{N}, x_{n 2}^{N}, \ldots, x_{n K}^{N}\right\}$, each vector representing $N$ nodal values mass fraction of the $n$th species at different times during the pulsing sequence This original data set $X_{n}$ is transformed to a new set of 
uncorrelated variables (POD modes)

$$
Z_{n}=\left\{z_{n 1}^{N}, z_{n 2}^{N}, \ldots, z_{n K}^{N}\right\}=X_{n} \Phi_{n},
$$

where the columns of $\Phi_{n}=\left\{\phi_{n 1}^{K}, \phi_{n 2}^{K}, \ldots, \phi_{n K}^{K}\right\}$ are the eigenvectors of the product matrix $\left(X_{n}{ }^{\prime} X_{n}\right) \phi_{n i}^{K}=\lambda_{n i} \phi_{n i}^{K}$, ranked, in descending order, with respect to the associated eigenvalue. The prime superscript denotes the transpose of the matrix. The POD modes $Z_{n}$ are orthogonal $z_{n i}^{N} \cdot z_{n j}^{N}=\lambda_{n i} \delta_{i, j}$, and the transformation of variables preserves the data variability

$$
\sum_{k=1}^{K}\left(X_{n}{ }^{\prime} X_{n}\right)_{k k}=\sum_{k=1}^{K}\left(Z_{n}{ }^{\prime} Z_{n}\right)_{k k}=\sum_{k=1}^{K} \lambda_{n k}
$$

Expansion of the original data $X_{n}$ in terms of the most significant POD modes minimizes the mean square error of a reduced basis representation [20]

$$
\left(X_{n}^{M}\right)_{i j}=\sum_{k=1}^{M}\left(Z_{n}\right)_{i k}\left(\Phi_{n}\right)_{k j}^{\prime}
$$

where $M<K$. The most significant POD modes are those corresponding to the largest eigenvalues, since the ratio of an eigenvalue to the summation of eigenvalues, $\lambda_{n k} / \sum_{j=1}^{K} \lambda_{n j}$, gives the percentage of the mean square error unaccounted for by eliminating the corresponding POD mode $z_{n k}^{N}[20]$ in the reduced basis representation.

\subsection{The Control Problem}

We seek to control the mass fractions of TMI and phosphine at the inlet $\left(\Gamma_{1}\right)$ of the reactor in order to obtain a desired flux $q_{T}(t)$ of reactants at point $\vec{x}_{p}$ on the susceptor $\left(\Gamma_{5}\right)$. The general flux at a point $\vec{x}_{p}$ is given by

$$
q(t)=\left[\begin{array}{c}
q_{I n}(t) \\
q_{P}(t)
\end{array}\right]=-\rho\left[\begin{array}{c}
\left.D_{1} \frac{W_{I n}}{W_{1}} \frac{\partial Y_{1}}{\partial n}\right|_{\vec{x}_{p}}+\left.D_{3} \frac{W_{I n}}{W_{3}} \frac{\partial Y_{3}}{\partial n}\right|_{\vec{x}_{p}} \\
\left.D_{2} \frac{W_{P}}{W_{2}} \frac{\partial Y_{2}}{\partial n}\right|_{\vec{x}_{p}}
\end{array}\right],
$$

where $W_{I n}$ and $W_{P}$ are the molecular weights of indium (a component of $Y_{1}$ and $Y_{3}$ ) and phosphorus $\left(Y_{2}\right)$, respectively.

Under the dilute approximation, the steady state solutions for $\vec{v}, \rho$, and $T$ can be used 
in (5). The full Dirichlet boundary control problem can then be formulated as

$$
\begin{array}{rlrl}
\frac{\partial Y_{n}}{\partial t}+\vec{v} \cdot \vec{\nabla} Y_{n} & =\frac{1}{\rho} \vec{\nabla} \cdot\left(\rho D_{n} \vec{\nabla} Y_{n}\right)+\lambda_{n} Y_{1} & \\
Y_{n}(0, \vec{x}) & =y_{n 0}(\vec{x}) & & \\
Y_{1}(t, \vec{x}) & =u_{1}(t) & & \text { on } \Gamma 1 \\
Y_{2}(t, \vec{x}) & =u_{2}(t) & & \text { on } \Gamma 1 \\
Y_{3}(t, \vec{x}) & =0 & & \text { on } \Gamma 1 \\
Y_{n}(t, \vec{x}) & =0 & & \text { on } \Gamma 5 \\
\frac{\partial Y_{n}(t, \vec{x})}{\partial n} & =0 & & \text { on } \Gamma 2 \\
n & =1,2,3 &
\end{array}
$$

where $Y_{1}, Y_{2}$, and $Y_{3}$ refer to the mass fractions of TMI, phosphine, and MMI, respectively, and $u_{1}(t), u_{2}(t)$ are the controls corresponding to TMI and phosphine, $\lambda_{1}(T)=$ $-k_{0} e^{(-E / R T)}, \lambda_{2}(T)=0$, and $\lambda_{3}(T)=\left(W_{3} / W_{1}\right) k_{0} e^{(-E / R T)}$.

We consider a finite time horizon problem of minimizing the cost function

$$
J(u, y)=\int_{0}^{T}\left[u^{\prime} R u+\left(q-q_{T}\right)^{\prime} Q\left(q-q_{T}\right)\right] d t
$$

where the flux $q(t)$ tracks the desired flux $q_{T}(t)$.

Penalty Boundary Formulation. We use a penalty boundary formulation of the timedependent species equations (5) to describe the transport of TMI, phosphine, and MMI in the reactor for implementation of the control problem

$$
\begin{array}{rlrl}
\frac{\partial Y_{n}}{\partial t}+\vec{v} \cdot \vec{\nabla} Y_{n} & =\frac{1}{\rho} \vec{\nabla} \cdot\left(\rho D_{n} \vec{\nabla} Y_{n}\right)+\lambda_{n} Y_{1} & \\
Y_{n}(0, \vec{x}) & =y_{n 0}(\vec{x}) & \\
\frac{\partial Y_{1}(t, \vec{x})}{\partial n} & =\frac{1}{\epsilon}\left(Y_{1}(t, \vec{x})-u_{1}(t)\right) & & \text { on } \Gamma 1 \\
\frac{\partial Y_{2}(t, \vec{x})}{\partial n} & =\frac{1}{\epsilon}\left(Y_{2}(t, \vec{x})-u_{2}(t)\right) & & \text { on } \Gamma 1 \\
\frac{\partial Y_{3}(t, \vec{x})}{\partial n} & =\frac{1}{\epsilon} Y_{3}(t, \vec{x}) & & \text { on } \Gamma 1 \\
\frac{\partial Y_{n}(t, \vec{x})}{\partial n} & =\frac{1}{\epsilon} Y_{n}(t, \vec{x}) & & \text { on } \Gamma 5 \\
\frac{\partial Y_{n}(t, \vec{x})}{\partial n} & =0 & & \text { on } \Gamma 2 \\
n & =1,2,3 &
\end{array}
$$

where, as before, $Y_{1}, Y_{2}$, and $Y_{3}$ refer to the mass fractions of TMI, phosphine, and MMI, respectively, and $u_{1}(t), u_{2}(t)$ are the controls. Under sufficient regularity, one can argue that solutions of (14), in the limit as $\epsilon \rightarrow 0$, approximate solutions for the problem 
with conditions $Y_{n}(t, \vec{x})=u_{n}(t)$ on $\Gamma 1$, and $Y_{n}(t, \vec{x})=0$ at $\Gamma 5$ (see $[5,12]$ for related discussions). A value of $\epsilon=1 \times 10^{-3}$ is used for the results presented here.

Writing (14) in weak form with test functions $w_{j}$, we obtain

$$
\begin{aligned}
\int_{\Omega} \frac{\partial Y_{n}}{\partial t} w_{j} d \Omega= & -\int_{\Omega}\left(\vec{v} \cdot \vec{\nabla} Y_{n}\right) w_{j} d \Omega-\int_{\Omega} D_{n} \vec{\nabla} Y_{n} \cdot \vec{\nabla} w_{j} d \Omega+\int_{\Omega} \frac{1}{\rho} w_{j} D_{n} \vec{\nabla} Y_{n} \cdot \vec{\nabla} \rho d \Omega \\
& +\int_{\Omega} \lambda_{n} Y_{n} w_{j} d \Omega+\frac{1}{\epsilon} \int_{\Gamma 1, \Gamma 5} w_{j} D_{n} Y_{n} d s-\frac{1}{\epsilon} \int_{\Gamma 1} w_{j} D_{n} u_{n} d s
\end{aligned}
$$

where $n=1,2,3$ and $u_{3} \equiv 0$. Spatially dependent values for $\vec{v}, T, \rho$, and $D_{n}$ are interpolated from the nodal values obtained from the CFD simulations.

Discretization Method I: finite elements. Two discretization formulations are applied to (15). The first formulation, a finite element approximation, produces the full system (which plays the role of a reactor simulator). In this case, the species mass fractions (15) are approximated using standard finite element discretization with $N=453$ quadratic interpolation functions $\psi_{i}$ and $N$ nodal coefficients $y_{n i}^{N}$

$$
Y_{n}^{N}(t, \vec{x})=\sum_{i=1}^{N} y_{n i}(t) \psi_{i}(\vec{x})
$$

Choosing the test functions to be $w_{j}=\psi_{j}, j=1,2, \ldots, N$, we obtain in a standard way the matrix equation

$$
\dot{y}^{3 N}(t)=A^{3 N} y^{3 N}(t)+B^{3 N} u(t) \quad u(t)=\left[\begin{array}{l}
u_{1}(t) \\
u_{2}(t)
\end{array}\right],
$$

where $A^{3 N}$ is an $3 N \times 3 N$ matrix, $B^{3 N}$ is a $3 N \times 2$ matrix, and the control $u$ is a control vector.

Discretization Method II: POD modes. The second discretization formulation produces the reduced basis model. In this case, we first use the POD modes $\left\{z_{n k}^{N}\right\}$ to obtain the POD basis elements

$$
\Psi_{n k}(\vec{x})=\sum_{i=1}^{N}\left(z_{n k}\right)_{i} \psi_{i}(\vec{x}), \quad k=1,2, \ldots, K,
$$

where the functions $\psi_{i}(\vec{x})$ are the finite element quadratic interpolation functions and $n=1,2,3$. The mass fraction for the $n$th species is approximated as a linear combination of the POD basis elements corresponding to the $M_{n}$ most significant POD modes

$$
Y_{n}^{M_{n}}(t, \vec{x})=\sum_{i=1}^{M_{n}} y_{n i}(t) \Psi_{n i}(\vec{x})
$$


where, in this case, $M_{n}<<K<<N$. Application of this approximation to (15) (in this case we use POD test functions $\left.w_{i}=\Psi_{n i}, i=1,2, \ldots, M\right)$ yields

$$
\dot{y}^{M}(t)=A^{M} y^{M}(t)+B^{M} u(t) \quad u(t)=\left[\begin{array}{l}
u_{1}(t) \\
u_{2}(t)
\end{array}\right],
$$

where $M=\sum_{n=1}^{3} M_{n}, A^{M}$ is an $M \times M$ matrix, and $B^{M}$ is an $M \times 2$ matrix.

Tracking Control. To control the reduced order system (20), we observe the flux $q(t)$ of indium and phosphorus, to the center of the substrate at $\vec{x}_{p}(11)$. The fluxes are approximated as

$$
q^{M}(t)=-\rho\left[\begin{array}{c}
\left.D_{1} \frac{W_{I n}}{W_{1}} \sum_{k=1}^{M_{1}} \frac{\partial \Psi_{1 k}}{\partial n}\right|_{\vec{x}_{p}} y_{1 k}(t)+\left.D_{3} \frac{W_{I n}}{W_{3}} \sum_{k=1}^{M_{3}} \frac{\partial \Psi_{3 k}}{\partial n}\right|_{\vec{x}_{p}} y_{3 k}(t) \\
\left.D_{2} \frac{W_{P}}{W_{2}} \sum_{k=1}^{M_{2}} \frac{\partial \Psi_{2 k}}{\partial n}\right|_{\vec{x}_{p}} y_{2 k}(t)
\end{array}\right]
$$

We seek the optimal control $u^{*}$ for (20) such that the output $q^{M}$ tracks a signal $q_{T}$ (the desired flux at $\vec{x}_{p}$ ), minimizing the generalized performance index (e.g., see $\left.[3,36]\right)$

$$
J\left(y_{0}, u(\cdot)\right)=\int_{0}^{\infty}\left[u^{\prime} R u+\left(\bar{q}^{M}\right)^{\prime} Q_{2} \bar{q}^{M}+\left(q^{M}-q_{T}\right)^{\prime} Q_{1}\left(q^{M}-q_{T}\right)\right] d t,
$$

where $Q_{2}$ keeps $\bar{q}^{M}$ bounded, $\bar{q}^{M}=\left(\bar{H}^{M}\right)^{\prime} y^{M},\left(\bar{H}^{M}\right)^{\prime}=I-L^{M}\left(H^{M}\right)^{\prime}$, $L^{M}=H^{M}\left(\left(H^{M}\right)^{\prime} H^{M}\right)^{-1}$, and $I$ is the identity matrix. Choosing $Q_{2}=r_{2} I, \quad R=I$, and

$$
Q_{1}=\left[\begin{array}{ll}
r_{11} & 0 \\
0 & r_{22}
\end{array}\right]
$$

we can rewrite this performance index as

$$
J\left(y_{0}, u(\cdot)\right)=\int_{0}^{\infty}\left[u^{\prime} R u+\left(y^{M}-y_{T}^{M}\right)^{\prime} Q\left(y^{M}-y_{T}^{M}\right)\right] d t,
$$

where $r_{11}, r_{22}$, and $r_{2}$ are design parameters, $Q=\overline{H^{M}} Q_{2}\left(\overline{H^{M}}\right)^{\prime}+H^{M} Q_{1}\left(H^{M}\right)^{\prime}$, and

$$
y_{T}^{M}(t)=L q_{T}(t)
$$

is the desired state trajectory. This is a standard formulation for a 'tracking' control problem $[3,36]$ for which a complete theory is known (for a summary and references see 
Chapter 7 of [8]). For the pulsed sources we specify time dependent target fluxes such that the output $q^{M}$ tracks the signal

$$
q_{T}(t)=\left[\begin{array}{l}
q_{T 1} f_{1}(t) \\
q_{T 2} f_{2}(t)
\end{array}\right],
$$

with constants $q_{T 1}, q_{T 2}$ and target flux profiles $f_{1}(t)$ and $f_{2}(t)$ (Fig. 3).

The optimal control is given by

$$
\begin{aligned}
u^{*} & =\left[\begin{array}{l}
u_{1}^{*}(t) \\
u_{2}^{*}(t)
\end{array}\right] \\
& =-K^{M} y^{M}-R^{-1}\left(B^{M}\right)^{\prime} b(t),
\end{aligned}
$$

where the gain is given by (see p84-85 of [3], [36], or [8])

$$
K^{M}=R^{-1}\left(B^{M}\right)^{\prime} \Pi
$$

and $\Pi$ satisfies the algebraic Riccati equation (ARE)

$$
0=\Pi A^{M}+\left(A^{M}\right)^{\prime} \Pi-\Pi B^{M} R^{-1}\left(B^{M}\right)^{\prime} \Pi+Q .
$$

The tracking variable $b(t)$ is given by

$$
\dot{b}(t)=-\left(A^{M}-B^{M} K^{M}\right)^{\prime} b(t)+Q y_{T}^{M}(t), \quad b(T)=0,
$$

where $T=T_{p}+\Delta, T_{p}$ is the total elapsed time for the TMI (or phosphine) pulse cycle, and $\Delta$ is five times the dominant time constant associated with the eigenvalues of $A^{M}-$ $B^{M} K^{M}$ (see p86 of [3]). Since there is a delay between application of the control (mass fraction of sources at the inlet) and detection of the corresponding signal (flux at the center of the substrate), the time dependent tracking variable plays a crucial role in the optimal control, by allowing it to anticipate and follow the time varying target fluxes.

State Estimation. Application of the tracking control to the reduced order model (20) yields

$$
\begin{aligned}
\dot{y}^{M} & =A^{M} y^{M}-B^{M} K^{M} y^{M}-B^{M} R^{-1}\left(B^{M}\right)^{\prime} b(t) \\
q(t) & =\left(H^{M}\right)^{\prime} y^{M}(t)
\end{aligned}
$$

with $K^{M}$ and $b(t)$ defined in (28) and (30), respectively. Note that this formulation requires complete reduced order state feedback for the gain $K^{M}$ of (28). Since the full state of the system (or even the reduced order system) in the reactor can not be observed, we implement a state estimator or compensator design based on observation (21) of the 


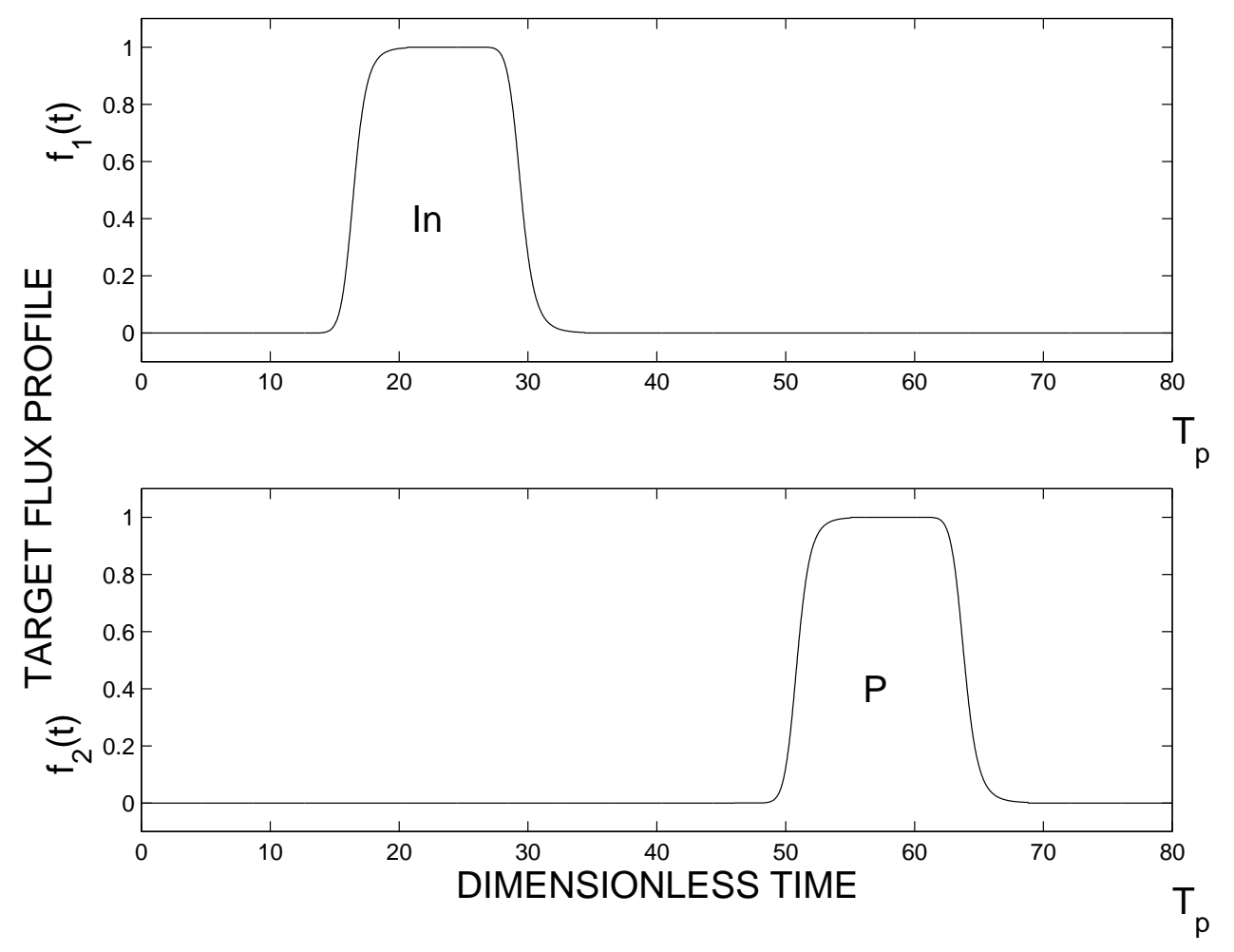

Figure 3: Indium and phosphorus target flux profiles.

flux at the center of the substrate. This yields (see [36] or Chapter 8 of [8] and the references therein) the coupled system

$$
\begin{aligned}
\dot{y}^{M} & =A^{M} y^{M}-B^{M} K^{M} y_{e}^{M}-B^{M} R^{-1}\left(B^{M}\right)^{\prime} b(t) \\
\dot{y}_{e}{ }^{M} & =A_{c} y_{e}^{M}+F^{M}\left(H^{M}\right)^{\prime} y^{M}-B^{M} R^{-1}\left(B^{M}\right)^{\prime} b(t),
\end{aligned}
$$

where $y_{e}^{M}$ is the $M \times 1$ vector approximation to the approximate state $y^{M}$, the compensator system operator $A_{c}$ is given by

$$
A_{c}=A^{M}-F^{M}\left(H^{M}\right)^{\prime}-B^{M} K^{M},
$$

and the compensator gain (Kalman filter) is given by

$$
F^{M}=\Sigma H^{M} V^{-1} .
$$

The matrix $\Sigma$ satisfies the dual ARE given by

$$
A^{M} \Sigma+\Sigma\left(A^{M}\right)^{\prime}-\Sigma H^{M} V^{-1}\left(H^{M}\right)^{\prime} \Sigma+U=0,
$$

where $U$ and $V$ are symmetric positive semi-definite and symmetric positive definite matrix design parameters, respectively. We choose $U=I$ and $V=r_{3}$, where $r_{3}$ is a third parameter at our disposal in designing the overall feedback control/compensator system. 
This implementation of the state estimator yields the following closed-loop system for 'optimal' control of the reduced order model

$$
\begin{aligned}
\left(\begin{array}{c}
\dot{y}^{M} \\
\dot{y}_{e}^{M}
\end{array}\right)= & \left(\begin{array}{cc}
A^{M} & -B^{M} K^{M} \\
F^{M}\left(H^{M}\right)^{\prime} & A^{M}-B^{M} K^{M}-F^{M}\left(H^{M}\right)^{\prime}
\end{array}\right)\left(\begin{array}{c}
y^{M} \\
y_{e}^{M}
\end{array}\right) \\
& -\left(\begin{array}{c}
B^{M} R^{-1}\left(B^{M}\right)^{\prime} b(t) \\
B^{M} R^{-1}\left(B^{M}\right)^{\prime} b(t)
\end{array}\right)
\end{aligned}
$$

with the optimal control

$$
u^{*}=-K^{M} y_{e}^{M}-R^{-1}\left(B^{M}\right)^{\prime} b(t) .
$$

Control of the Full System. Use of the design given in (36) and (37) can be expected to produce a stabilized and generally efficient system for control of the reduced order model (20). However, this is not the issue of practical importance in our efforts. The goal, of course, is to design a feedback control/compensator system for (14) (or in weak form (15)), which we hope will be a good approximation (for proper choice of $\epsilon$ ) for the actual physical dynamics (1)-(5), i.e., transient solutions of (5) with (1)-(4) in steady state. Thus, the real measure of the value of the control/compensator system designed above via the reduced order model is how well it performs when used in the actual physical system. Short of applying the control/compensator system to the physical reactor in experiments, our best assessment of its utility is when applied to the 'full' system (17). That is, we should computationally test the reduced order control/compensator design based on (28)-(30), (33)-(37) in the system (17).

Application of the reduced order tracking control to the full system with the reduced order state estimator yields

$$
\begin{aligned}
\left(\begin{array}{c}
\dot{y}^{N} \\
\dot{y}_{e}^{M}
\end{array}\right)= & \left(\begin{array}{cc}
A^{N} & -B^{N} K^{M} \\
F^{M}\left(H^{N}\right)^{\prime} & A^{M}-B^{M} K^{M}-F^{M}\left(H^{M}\right)^{\prime}
\end{array}\right)\left(\begin{array}{c}
y^{N} \\
y_{e}^{M}
\end{array}\right) \\
& -\left(\begin{array}{c}
B^{N} R^{-1}\left(B^{M}\right)^{\prime} b(t) \\
B^{M} R^{-1}\left(B^{M}\right)^{\prime} b(t)
\end{array}\right)
\end{aligned}
$$

where $H^{N}$ is the observation vector for the full-dimensional system $q^{N}=\left(H^{N}\right)^{\prime} y^{N}(t)$, and $H^{M}, K^{M}, g^{M}$, and $F^{M}$ are as defined in (21), (28), (30), and (34), respectively. The optimal control is given by (37). We report on our simulations for system (38) with different values of the design parameters $r_{11}$ and $r_{22}$ in the results of Section 3. Values of $r_{2}$ and $r_{3}$ were varied and then fixed at nominal performance values in the calculations reported below.

Implementation. The governing equations (14) are nondimensionalized prior to the calculation of the coefficient matrices $\left(L_{0}=1 \times 10^{-2} \mathrm{~m}, D_{0}=1.15 \times 10^{-3} \mathrm{~m}^{2} / \mathrm{s}\right.$, and 
$\left.\rho_{0}=4.04 \times 10^{-2} \mathrm{~kg} / \mathrm{m}^{3}\right)$. The POD modes and the coefficient matrices $A^{N}, B^{N}, H^{N}$, $A^{M}, B^{M}$, and $H^{M}$ in (38) are calculated using in-house C programs. All other matrix calculations are implemented in the Matlab computing environment (The Math Works Inc., Natick, MA); the optimal gain matrix $K^{M}$ and Riccati solution $\Pi$ are determined using Matlab's lqr() function.

The dynamical equations are integrated using a semi-implicit extrapolation integration method for stiff equations with adaptive step size control (stifbs(), and $\operatorname{simpr}()$ in [35]). During the clearance cycles the optimal control (mass fractions at the inlet) may become negative. Since negative mass fractions are not physically meaningful, we implement a truncation procedure at each step of the integration, which sets negative control values equal to zero. The resulting control is suboptimal, but it will be seen that control authority is maintained. 


\section{Results}

We report in sequential form results from the series of computations and simulations described in detail in Section 2.

\subsection{CFD Simulation Results}

We first report on results of the CFD package simulations described in Section 2.1. Contour plots of the steady-state solutions of the temperature and the x-component of the velocity (Fig. 4a-b) give an indication of the steady-state transport conditions in the reactor. With the top wall and inlet maintained at room temperature, there is a steep temperature gradient (Fig. 4a) upstream from the substrate. In the region above the substrate, the isotherms run parallel to the hot substrate and the opposite cold wall. The velocity of the gas increases more than 4 -fold as it passes in the vicinity of the hot substrate (Fig. 4b), while the y-component of the velocity (not shown) remains small throughout the reactor.

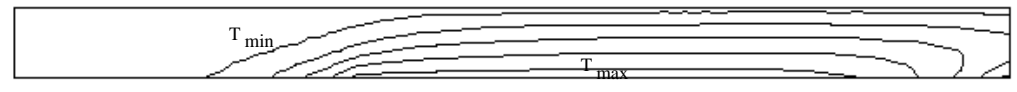

(a) Temperature

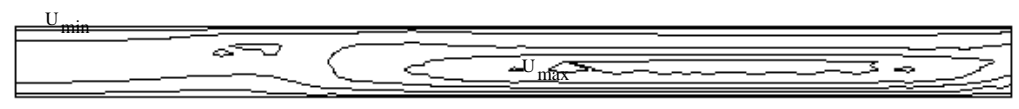

(b) X-component of velocity

Figure 4: Contour plot of steady-state values for the (a) temperature in $100^{\circ} \mathrm{K}$ steps with $\mathrm{T}_{\max }=750^{\circ} \mathrm{K}$ and $\mathrm{T}_{\min }=350^{\circ} \mathrm{K}$, (b) $\mathrm{x}$-component of the velocity in $0.1 \mathrm{~m} / \mathrm{s}$ steps with $\mathrm{v}_{\max }=0.45$ and $\mathrm{v}_{\min }=0.05$ $\mathrm{m} / \mathrm{s}$. 
A plot of the flux of In and $\mathrm{P}$ at the substrate center as a function of time (Fig. 5) shows that the flux plateaus approximately $1 \mathrm{~s}$ after the reactant is introduced into the reactor and is not completely cleared from the reactor until one second after the cessation of the reactant pulse.

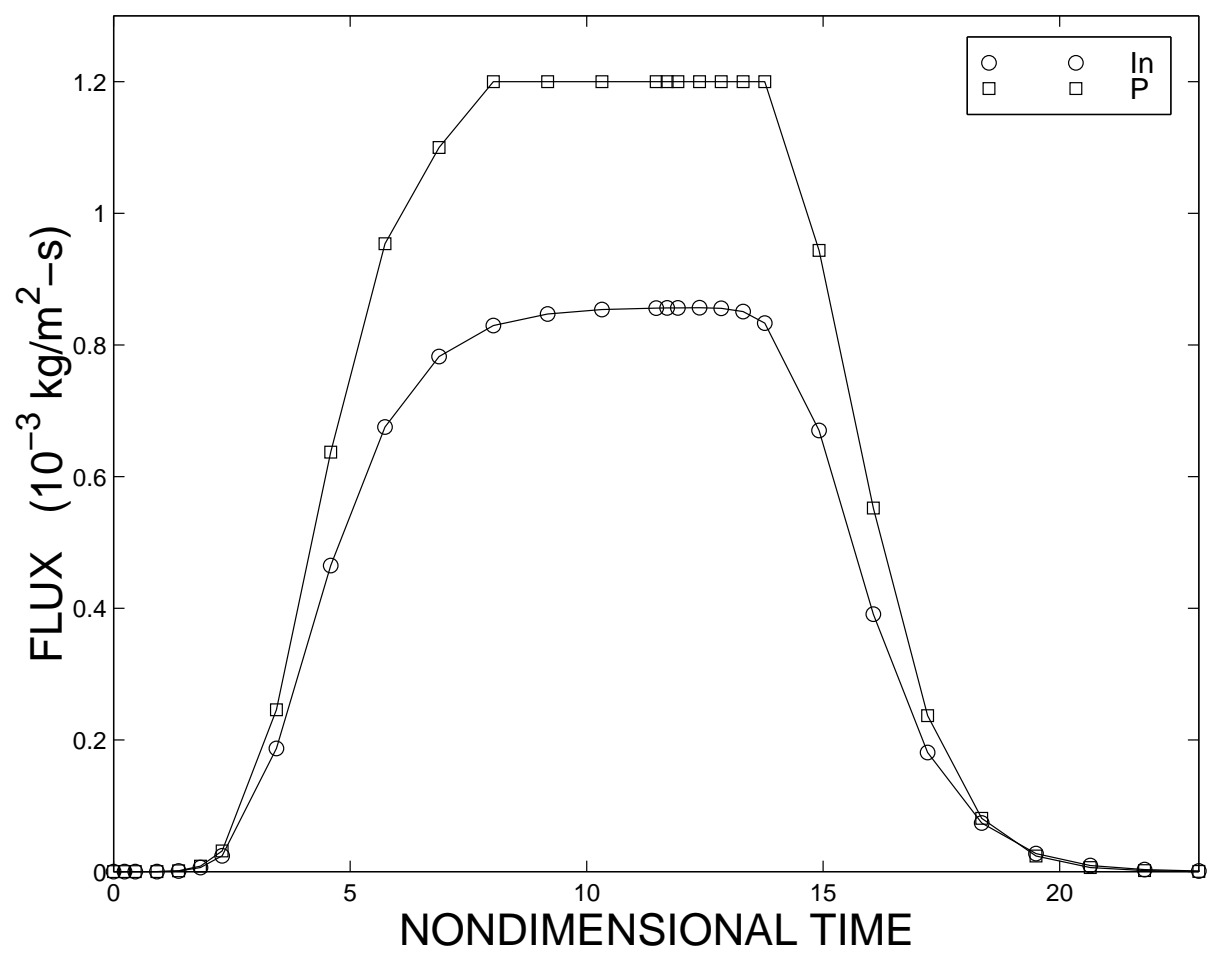

Figure 5: Flux of In and $\mathrm{P}$ to the substrate center as a function of time. 


\subsection{Construction of POD Modes}

The POD modes for each species are constructed from 150 snapshots $(K=150)$ taken during the three second cycle (2 s pulsing, $1 \mathrm{~s}$ clearing) of each source species. Each solution vector represents the species mass fraction at the 453 nodal points and corresponds to a time increment of 0.03-s in the time range from 0 to 3 seconds. A plot of the captured variability $\left(\sum_{j=1}^{M_{n}} \lambda_{n j} / \sum_{k}^{K} \lambda_{n k}\right)$ as a function of the number of modes $\left(M_{n} \leq 100\right)$ used (Fig. 6) shows that the original data is well-represented by 9 modes, or fewer. This strongly suggests using $M_{n} \leq 9$ in our reduced order model.

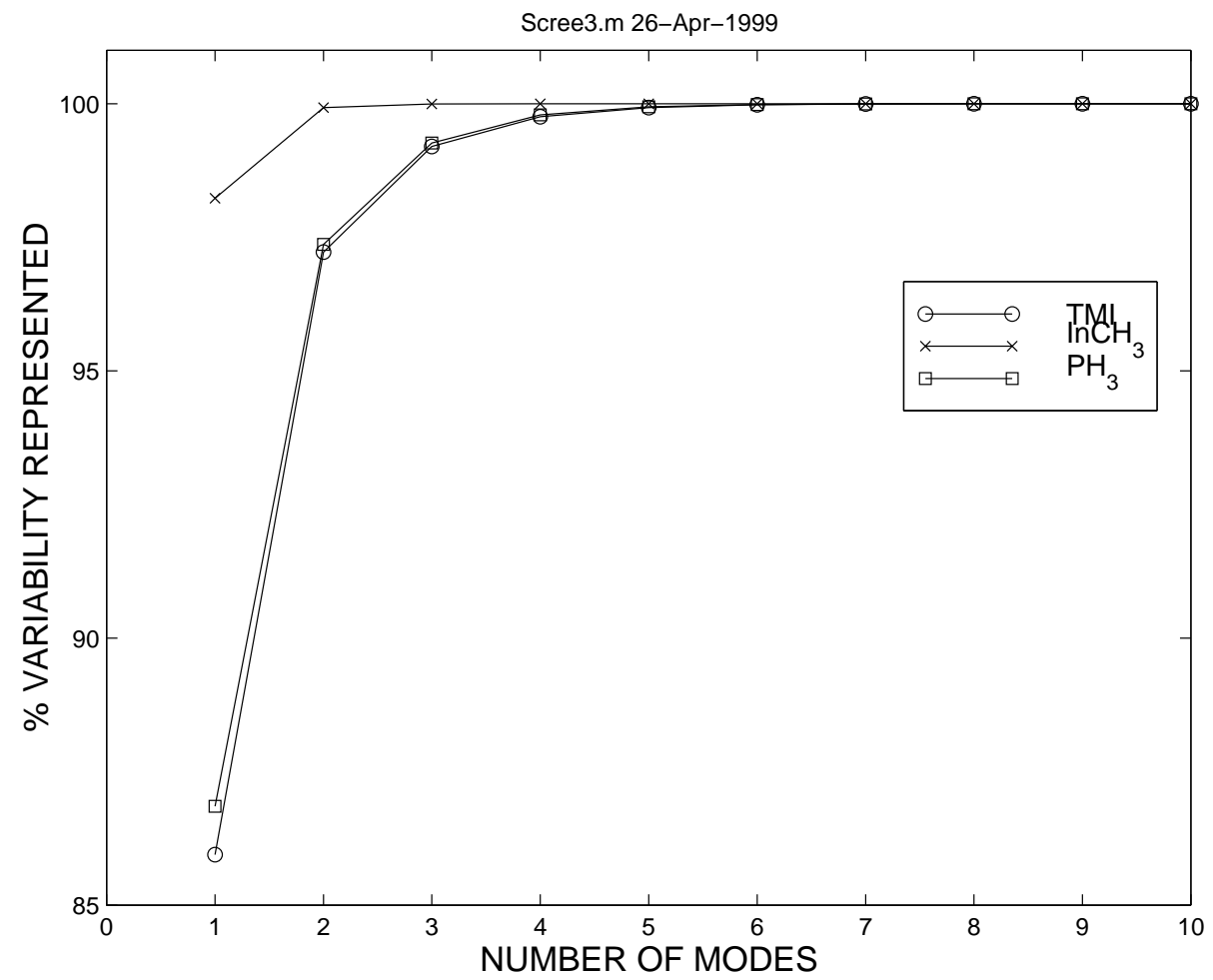

Figure 6: Total percent variability captured as a function of the number of modes for each species (TMI, MMI, phosphine).

While the above comments suggest the proper order for accurate reduced order model simulations, there are additional order questions related to the control system to be used in determining reduced order gains and compensators. The ranks of the controllability and observability matrices have sometimes been found to be useful criteria (see the discussion in $[9,10,26])$ to help in the choice of the number of modes to use in control design applications for the reduced basis representation (19). The controllability of the 
linear system (20) is determined from the rank of the controllability matrix $\mathcal{C}$, where

$$
\mathcal{C}\left(\mathrm{A}^{\mathrm{M}}, \mathrm{B}^{\mathrm{M}}\right)=\left[\mathrm{B}^{\mathrm{M}}\left|\mathrm{A}^{\mathrm{M}} \mathrm{B}^{\mathrm{M}}\right|\left(\mathrm{A}^{\mathrm{M}}\right)^{2} \mathrm{~B}^{\mathrm{M}}|\ldots|\left(\mathrm{A}^{\mathrm{M}}\right)^{\mathrm{M}-1} \mathrm{~B}^{\mathrm{M}}\right] .
$$

Using standard results from optimal control theory [17], the $M$-dimensional linear system (20) is controllable if, and only if, the rank of the controllability matrix is equal to $M$. Similarly, the rank of the observability matrix $\mathcal{O}$ indicates the observability of the linear system (31), where

$$
\mathcal{O}\left(\mathrm{A}^{\mathrm{M}}, \mathrm{H}^{\mathrm{M}}\right)=\left[\mathrm{H}^{\mathrm{M}}\left|\left(\mathrm{A}^{\mathrm{M}}\right)^{\prime} \mathrm{H}^{\mathrm{M}}\right|\left(\left(\mathrm{A}^{\mathrm{M}}\right)^{2}\right)^{\prime} \mathrm{H}^{\mathrm{M}}|\ldots|\left(\left(\mathrm{A}^{\mathrm{M}}\right)^{\mathrm{M}-1}\right)^{\prime} \mathrm{H}^{\mathrm{M}}\right] .
$$

For the system under investigation in this paper, ranks of the controllability and observability matrices are recorded in Table 1 as a function of the number of modes in the reduced order model. Based on these results, which suggest that the maximum ranks of the controllability and observability matrices are ten and twelve, respectively. However, we should remember that these ranks are for the reduced order system and we are applying the control in the full order system. Nonetheless, we first used ten POD modes ( $\left.M=10: M_{1}=4, M_{2}=4, M_{3}=2\right)$ in our control/compensator applications to construct our reduced order model (20) and obtained adequate control authority. With further experimentation, we found that by adding more modes ( $M=19: M_{1}=8, M_{2}=8, M_{3}=3$ ), though no longer being assured of the controllability and observability of the reduced order system, we were able to achieve tighter control and reduce the oscillations of the system as it tracks the desired flux values (see Fig. 9 below) in the full order system.

Table 1: Rank of the Controllability and Observability Matrices

\begin{tabular}{rcccccccc}
\hline \multicolumn{8}{c}{ Reduce Order Model } \\
\multicolumn{8}{c}{ Dimension M and \% Variability Represented } \\
\multicolumn{2}{c}{ TMI } & \multicolumn{2}{c}{ Phosphine } & \multicolumn{2}{c}{ MMI } & \multicolumn{2}{c}{ Rank } \\
$M_{1}$ & $\%$ & $M_{2}$ & $\%$ & $M_{3}$ & $\%$ & $\mathcal{C}$ & $\mathcal{O}$ \\
\hline 5 & $(99.930)$ & 5 & $(99.942)$ & 3 & $(99.995)$ & 10 & 12 \\
5 & $(99.930)$ & 5 & $(99.942)$ & 2 & $(99.929)$ & 10 & 12 \\
4 & $(99.756)$ & 4 & $(99.793)$ & 2 & $(99.929)$ & 10 & 10 \\
5 & $(99.930)$ & 4 & $(99.793)$ & 2 & $(99.929)$ & 10 & 11 \\
5 & $(99.930)$ & 3 & $(99.264)$ & 2 & $(99.929)$ & 9 & 10 \\
5 & $(99.930)$ & 4 & $(99.793)$ & 1 & $(98.230)$ & 10 & 10 \\
8 & $(99.999)$ & 8 & $(99.999)$ & 3 & $(99.995)$ & 10 & 12 \\
\hline
\end{tabular}




\subsection{Control of Full System Using the Reduced Order State Es- timator}

A set of representative results are depicted here, where the reduced order state estimator is used to control the full system (38) using non-dimensional tracking values of $q_{T 1}=$ 0.0855 and $q_{T 2}=0.15$. In this example, we fix the design parameters $r_{11}=5000$, $r_{2}=1 \times 10^{-6}$, and $r_{3}=1 \times 10^{4}$, and vary the design parameter $r_{22}=150,200$, or 300. Initially, the solution vector for the estimated state is given a small nonzero value $\left(y_{e}^{M}=1 \times 10^{-4}\right)$, while the full system solution is given an initial value of zero $\left(y^{3 N}=0\right)$, where $N=453$ and $M=19\left(M_{1}=8, M_{2}=8, M_{3}=3\right)$. The tracking values and pulse profiles were chosen so that the integrated quantity of material deposited represents approximately one monolayer each of indium and phosphorus (assuming a 100 surface with $4.1 \times 10^{18}$ sites per $\mathrm{m}^{2}[40]$ ). Because of the truncation employed (see Section 2.2) the results shown here are not optimal. However, we demonstrate that the reduced order state estimator or compensator system is still capable of substantial control authority when applied to the full system. 
The controls $\left(u_{1}(t), u_{2}(t)\right)$ are plotted in Fig. 7 as a function of time along with the (nondimensional) target flux pulse cycle (indicated by the dotted lines). From Fig. 7 it can be seen that the control rises and falls in advance of the tracking pulses. Recall that this is necessary because of the delay between introduction of reactant at the inlet and transport of reactant(s) to the substrate. It is also evident from Fig. 7 that the control values oscillate as the system adjusts to match the observed flux to the desired tracking flux. Both the peak values and the magnitude of the oscillations of $u_{2}$ (corresponding to the phosphine mass fraction at the inlet) increase as $r_{22}$ increases, i.e., as more weight is placed on achieving the desired flux and less weight is placed on the cost of control. As expected, the profile for $u_{1}$ (corresponding to the TMI mass fraction at the inlet) is unchanging since $r_{11}$ is held constant in this example.

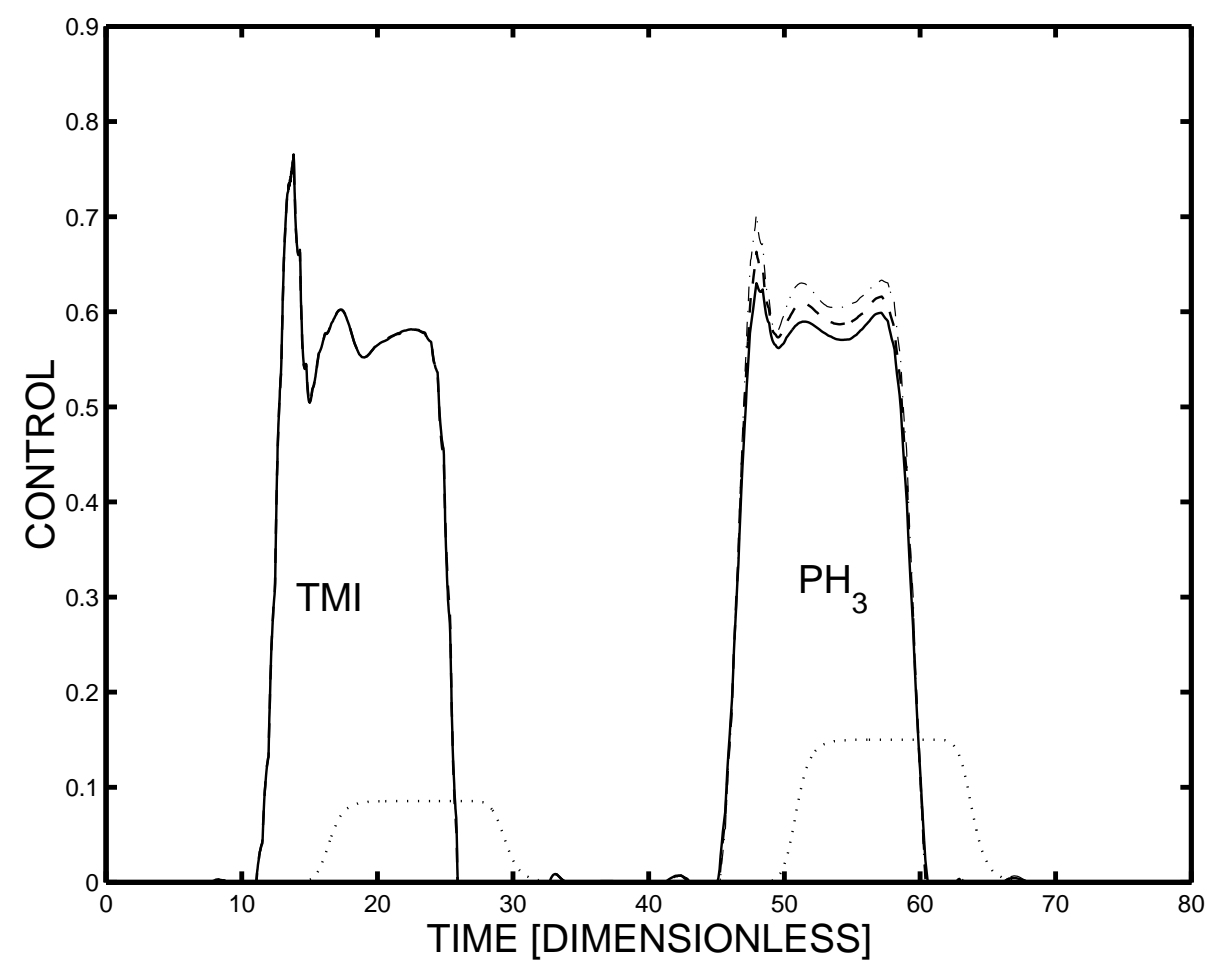

Figure 7: Control values (TMI and phosphine normalized mass fractions at the inlet) as a function of time for different values of the control parameter $r_{22}$ : 150 (solid line), 200 (dashed line), and 300 (dash-dot line). A nondimensional representation of the target flux profile is also shown (dotted line) for reference. The control rises and falls in advance of the tracking pulses. 
A plot of the observed flux as a function of time (Fig. 8) shows that the system is able to closely track the time dependence of the desired flux profile without significant delays. The ability of the system to match the target flux is sensitive to the design parameter $r_{22}$ with close agreement for the case of $r_{22}=200$.

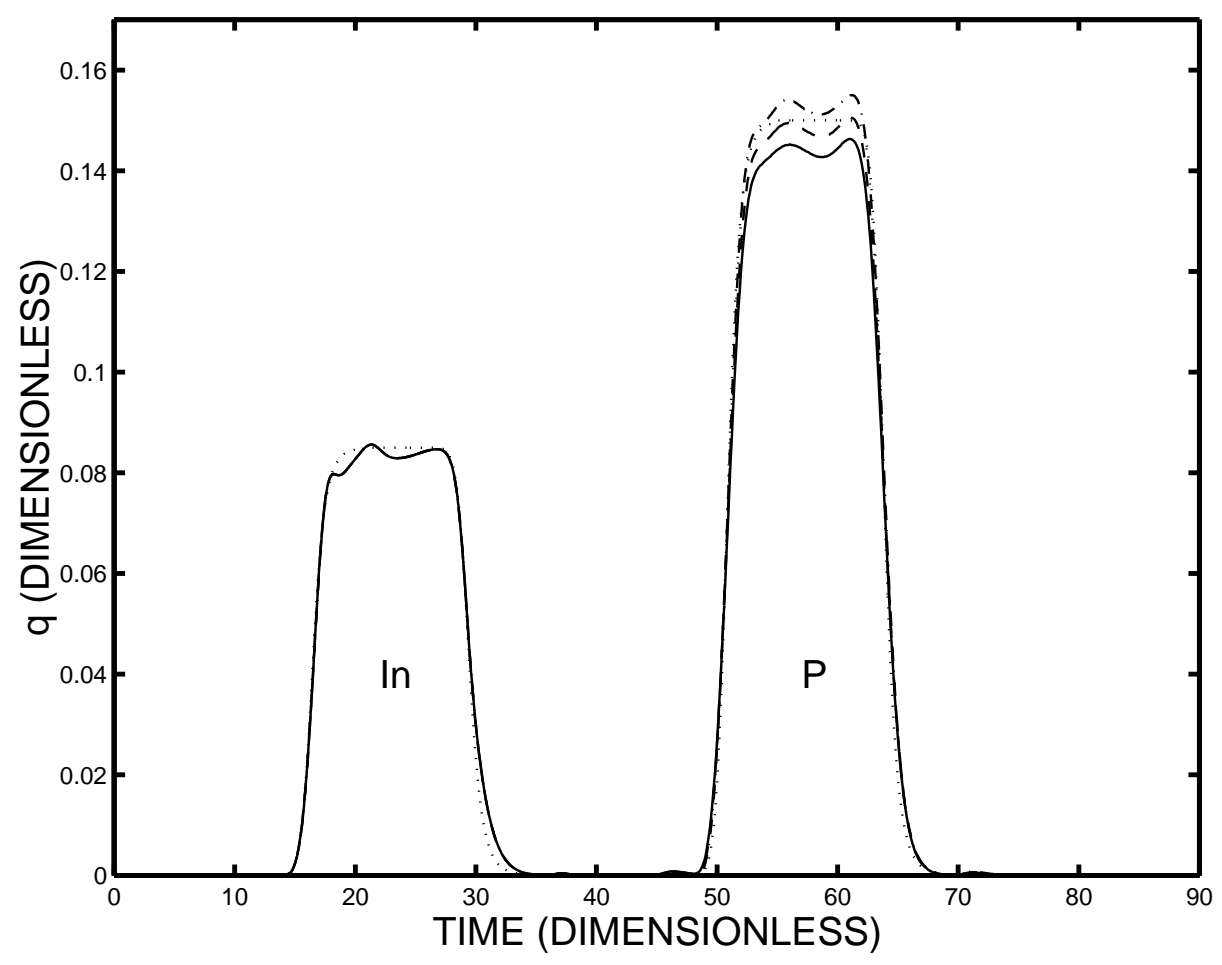

Figure 8: Observed fluxes as a function of time for different values of the control parameter $r_{22}: 150$ (solid line), 200 (dashed line), and 300 (dash-dot line). The target flux profile is also shown (dotted line) for reference. The system is able to closely track the desired flux profile.

Some oscillation of the observed flux can be seen in Fig. 8. The amplitudes of these oscillations increase with increasing control (larger values of $r_{22}$ or $r_{11}$ ) and increase as the size of the reduced order system is decreased. A comparison of the observed flux using two different reduced order dimensions (Fig. 9) shows that reducing the order from $M=19$ to $M=10$ (the apparent rank of the controllability matrix for the reduced order system) increases the amplitude of the oscillations.

The ability of the system to track the pulse turn off is limited when the target pulse fall off is steep. Since there is no mechanism for removing reactant from the system other than the carrier gas transport and absorption at the substrate, the observed flux can not fall off faster than some characteristic rate associated with the physical parameters of the problem (e.g., reactor dimension and carrier gas flow rate). As a result, the observed flux may have a tail that persists substantially beyond the target pulse drop. 


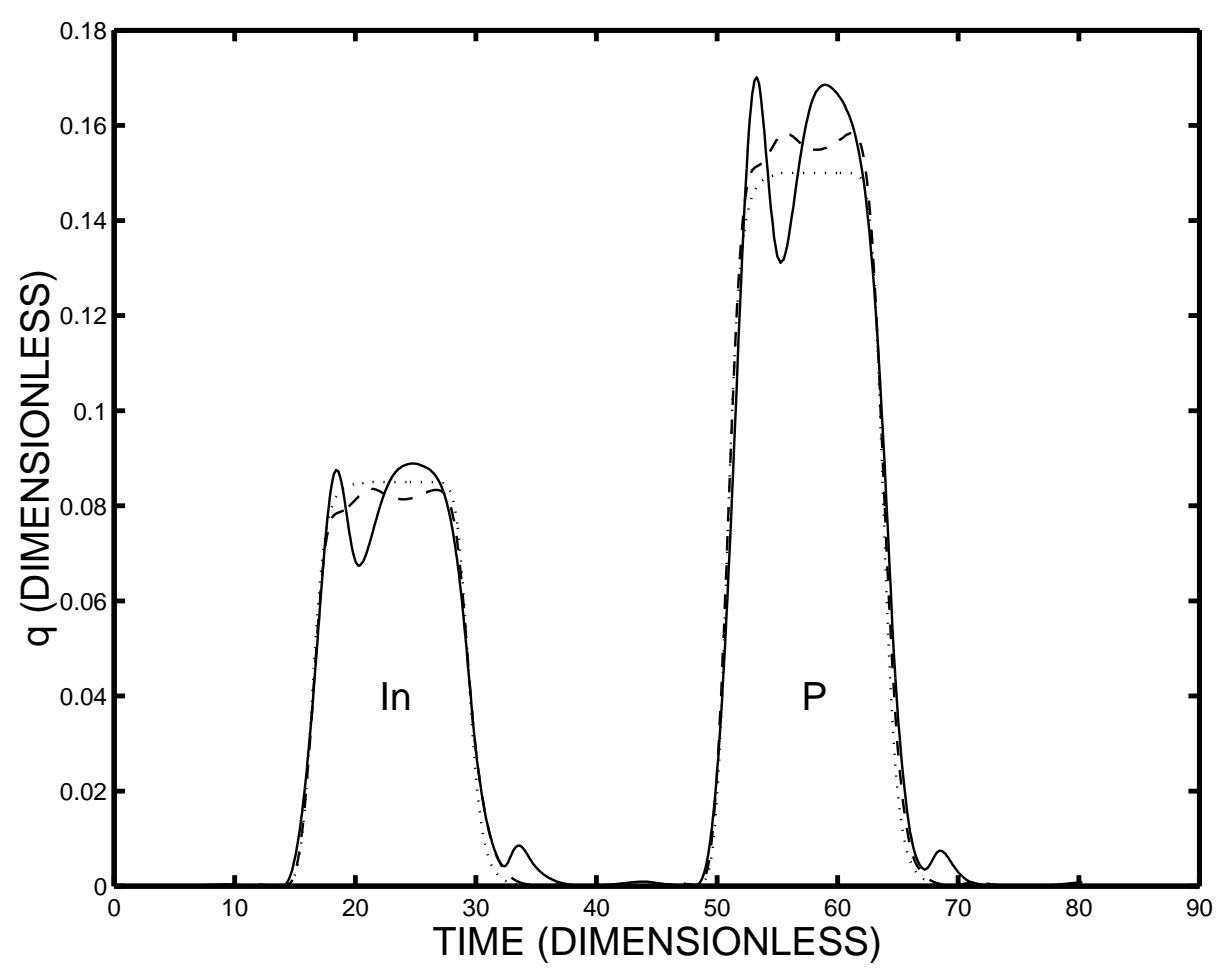

Figure 9: Comparison of observed flux as a function of time for two reduced order dimensions: $M=19$ (dashed line) and $M=10$ (solid line). The target flux profile is also shown (dotted line) for reference. The design parameter values for these simulations were as follows: $q_{T 1}=0.0855, q_{T 2}=0.15, r_{11}=1600$, $r_{22}=500, r_{2}=1 \times 10^{-4}$, and $r_{3}=1 \times 10^{4}$. The magnitude of the oscillations increases when the order is reduced.

Fig. 10 plots the norm of the difference between the full system solution and the state estimated solution as a function of time. The error decreases from an initial non-zero, the value of which depends on the initial conditions, then begins to rise as the control values rise in anticipation of the rising edge of the target flux pulse. The error decreases again as the target flux plateaus and the control values are relatively constant, then rises again as the control values fall in anticipation of the target flux drop off. The error reaches a minimum again during the clearance time period, after which the cycle of rising and falling is repeated again for the phosphine pulse. 


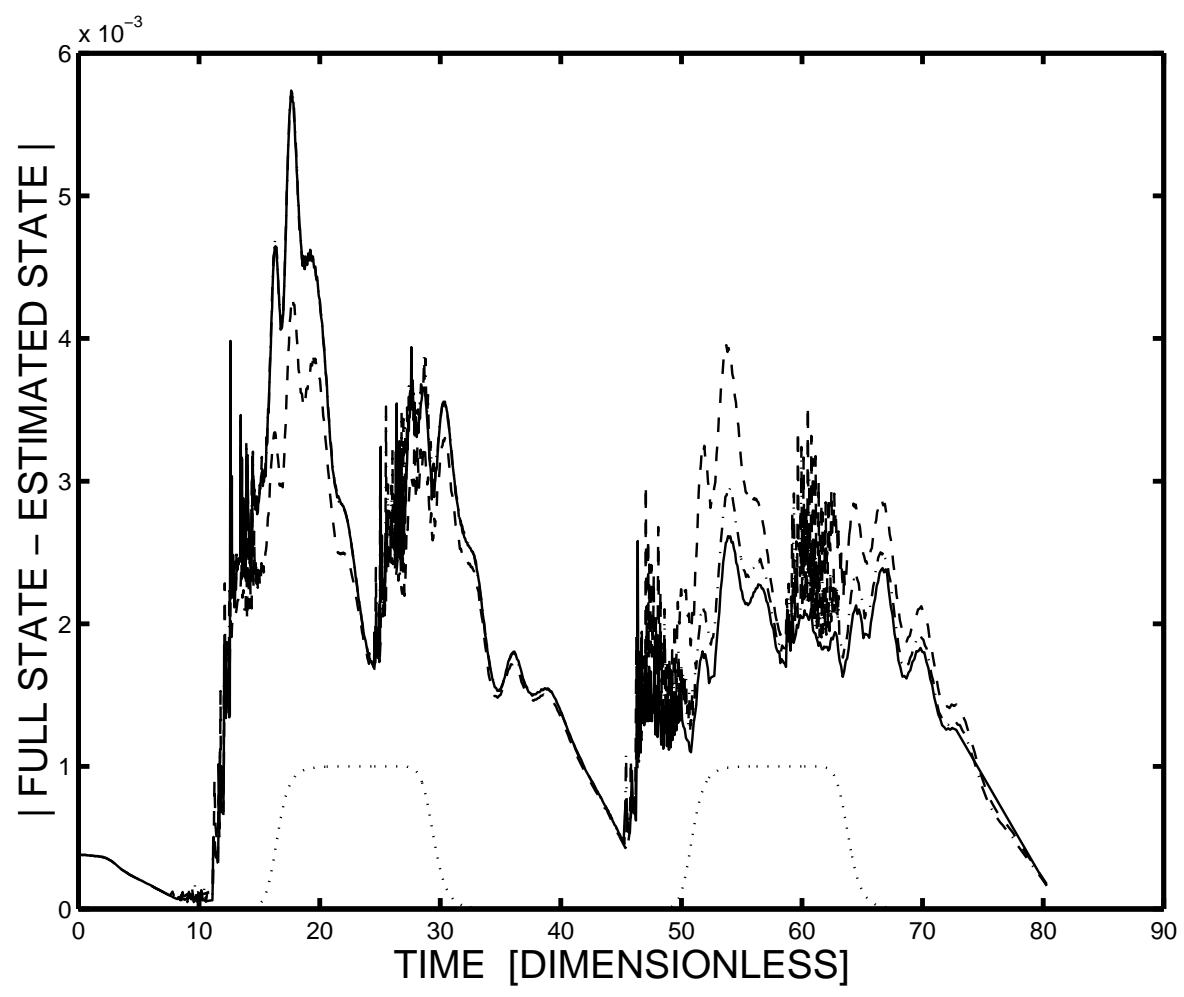

Figure 10: The norm of the difference between the full state and the POD estimated state for different values of the control parameter $r_{22}$ : 150 (solid line), 200 (dashed line), and 300 (dash-dot line). A nondimensional representation of the target flux profile is also shown (dotted line) for reference. The error rises as the control values change and decreases for during periods of (relatively) constant control.

\section{Conclusion}

We have demonstrated a computational implementation of reduced order feedback control of pulsed HPCVD III-V film growth involving the transport of multiple species with linear gas phase reactions. We implement feedback control using a reduced order state estimator based on observations of the fluxes of the Group III and Group V reactants at the substrate center. These observations are compatible with current PRS sensing technology. The controls are chosen so that the output fluxes track time dependent target fluxes, similar to the pulsed sources currently employed for HPCVD film growth. Because the control values must be constrained to be positive (positive mass fraction) the resulting truncated control is suboptimal, but the reduced order model design is still capable of substantial control authority.

Use of the POD-based design method allows us to reduce the order of the system with respect to a standard finite element representation, from $3 N=2159$ to $M=19$. These 
results suggest that real-time feedback control with partial state observations is a feasible goal for HPCVD reactors operating in steady state flow regimes with pulsed vapor reactant inputs. The positive results on this linear system suggest one possible approach for treating more complex situations that may be encountered when nonlinear gas phase or surface phase reactions are present: linearizing the equations about an optimal open loop solution and then using a reduced order feedback design on the linearized system.

\section{Acknowledgments}

This work was supported in part by DOD/AFSOR MURI Grant No. AFOSR-F4962095-1-0447. The authors are grateful to Dr. Klaus Bachmann and other members of the applied math/material sciences team at N.C. State for numerous helpful conversations during our control methodology development efforts, part of which are reported here. 


\section{References}

[1] J.A. Atwell, and B. King, Proper orthogonal decomposition for reduced basis feedback controllers for parabolic equations, ICAM Rep. 99-01-01, VPISU, Blacksburg VA, January 1999; Math. and Comput. Modeling, to appear.

[2] J.A. Atwell, and B. King, Reduced order controllers for spatially distributed systems via proper orthogonal decomposition, ICAM Rep. 99-07-01, VPISU, Blacksburg VA, July 1999.

[3] B.D.O. Anderson, and J.B. Moore, Optimal Control: Linear Quadratic Methods, (Prentice Hall, Englewood Cliffs, NJ, 1990).

[4] N. Aubry, P. Holmes, J.L. Lumley, and E. Stone, The dynamics of coherent structures in the wall region of a turbulent boundary layer, Journal of Fluid Mechanics 192, 115-173 (1988).

[5] I. Babuška, The finite element method with penalty, Mathematics of Computation 27, 221-228 (1973).

[6] K.J. Bachmann, N. Sukidi, C. Höpfner, C.Harris, N.A. Dietz, H.T. Tran, S. Beeler, K. Ito, and H.T. Banks. Real-time monitoring of steady-state pulsed chemical beam epitaxy by p-polarized reflectance, J. Crystal Growth 183, 323-337 (1998).

[7] K.J. Bachmann, H.T. Banks, C. Höpfner, G.M. Kepler, S.LeSure, S.D. McCall, and J.S. Scroggs, Optimal design of a high pressure organometallic chemical vapor deposition reactor, Mathematical and Computer Modelling (to appear).

[8] H.T. Banks, R.C. Smith, and Y. Wang, Smart Material Structures: Modeling, Estimation and Control, (Maisson/J.Wiley, Paris/Chichester, 1996).

[9] H.T. Banks, R.C.H. del Rosario, and R.C. Smith, Reduced order model feedback control design: Numerical implementation in a thin shell model, CRSC Tech Rep 98-27, N.C. State University, July 1998; IEEE Trans. Auto. Control, to appear.

[10] H.T. Banks, R.C.H. del Rosario, and R.C. Smith, Reduced order model feedback control design: Computational studies for thin cylindrical shells, CRSC Tech Rep 98-25, N.C. State University, June 1998.

[11] H.T. Banks, M.L. Joyner, B. Wincheski, and W.P. Winfree, Evaluation of material integrity using reduced order computational methodology, CRSC Tech Rep 99-30, N.C. State University, August 1999; Inverse Problems, submitted.

[12] J.W. Barrett and C.M. Elliot, Finite element approximation of the Dirichlet problem using the boundary penalty method, Numerische Mathematik 49, 343-366 (1986). 
[13] Y. Bayazitoglu and M.N. Ozisik, Elements of Mass Heat Transfer, (McGraw Hill, NY, 1988).

[14] G. Berkooz, Observations on the proper orthogonal decomposition, Studies in Turbulence, eds.: T.B. Gatski, S. Sarkar, and C.G. Speziale, (Springer-Verlag, New York, 1992), 229-247.

[15] G. Berkooz, P. Holmes, and J.L. Lumley, The proper orthogonal decomposition in the analysis of turbulent flows, Annual Review of Fluids Mechanics 25, N5:539-575, (1993).

[16] G. Berkooz, P. Holmes J.L. Lumley, and J.C. Mattingly, Low-dimensional models of coherent structures in turbulence, Physics Reports-Review Section of Physics Letters 287, N4:338-384 (1997).

[17] W.L. Brogan, Modern Control Theory, (Prentice Hall, NJ, 1991).

[18] D.H. Chambers, R.J. Adrian, P. Moin, D.S. Stewart, and H.J. Sung, Karhunen-Loève expansion of Burgers' model of turbulence, Phys. Fluids 31, 2573-2582 (1988).

[19] N. Dietz, V. Woods, K. Ito and I. Lauko, Real-time Optical Control of $\mathrm{Ga}_{1-x} \operatorname{In}_{x} \mathrm{P}$ Film Growth CRSC Tech Rep 99-26, N.C. State University, August 1999; Appl. Phys. Letters, submitted.

[20] K. Fukunaga, Introduction to Statistical Pattern Recognition, (Academic Press, NY, 1972).

[21] M. Gevelber, M. Toledo-Quiñones, and M. Bufano, Towards closed-loop control of CVD coating microstructures, Materials Science \& Engineering A 209, 377-383 (1996).

[22] A. Iollo, S. Lanteri, and J.A. Désidéri, Stability properties of POD-Galerkin approximations for the compressible Navier-Stokes equations, INRIA Rep. de Rech. no. 3589, December, 1998, Sophia Antipolis.

[23] J.E. Jackson, A User's Guide to Principal Components, (Wiley, NY, 1991).

[24] K. Karhunen, Zur spektral theorie stochasticher prozesse, Ann. Acad. Sci. Fennicae Ser. A1 Math Phys. 37, (1946).

[25] G.M. Kepler, C. Höpfner, J.S. Scroggs, and K.J. Bachmann, Feasibility of a vertical reactor for high pressure MOCVD, Materials Science \&f Engineering B 57, 9-17 (1998). 
[26] G.M. Kepler, H.T. Tran, and H.T. Banks, Reduced order model compensator control of species transport in a CVD reactor, CRSC Tech Rep 99-15, N.C. State University, April 1999; Optimal Control Applications and Methods, submitted.

[27] K. Kunisch and S. Volkwein, Control of Burgers' equation by a reduced order approach using proper orthogonal decomposition, Optimierung und Kontrolle Bericht Nr. 138, September, 1998, Universitat Graz, Austria; J. Opt. Theory Applic., to appear.

[28] K. Kunisch, and S. Volkwein, Galerkin proper orthogonal decomposition methods for parabolic systems, Sept. 1999, preprint.

[29] D.R. Lide and H.V. Kehiaian, CRC Handbook of Thermophysical and Thermochemical Data, (CRC Press, Boca Raton, 1994).

[30] M. Loève, Functions aleatoire de second ordre, Compte Rend. Acad. Sci. (Paris), 220 (1945).

[31] J.L. Lumley, The structure of inhomogeneous turbulent flows, in Atmospheric Turbulence and Radio Wave Propagation, A.M. Yaglom and V.I. Tatarski, eds., (Nauka, Moscow, 1967) 166-178.

[32] J.L. Lumley, Stochastic Tools in Turbulence, (Academic Press, New York, 1970).

[33] H.V. Ly and H.T. Tran, Proper orthogonal decomposition for flow calculations and optimal control in a horizontal CVD reactor, CRSC Tech Rep 98-13, N.C. State University, March 1998; Quarterly of Applied Mathematics, to appear.

[34] H.V. Ly and H.T. Tran, Modeling and control of physical processes using proper orthogonal decomposition, Computers and Mathematics with Applications, to appear.

[35] W.H. Press, S.A. Teukolsky, W.T. Vetterling, and B.P. Flannery, Numerical Recipes in C, 2nd edition, (Cambridge University Press, New York, 1994).

[36] D.L. Russell, Mathematics of Finite-Dimensional Control Systems: Theory and Design, (Marcel Dekker, New York, 1979).

[37] L. Sirovich and M. Kirby, Low-dimensional procedure for the characterization of human faces, J. Opt. Soc. Am. 4, 519-524 (1987).

[38] L. Sirovich, Chaotic dynamics of coherent structures, Physica D 37, 126-145 (1989).

[39] R.A. Svehla, NASA Technical Report R-132, 1962. 
[40] C. Theodoropolous, N.K. Ingle, T.J. Mountziaris, Z.-Y. Chen, P.L. Liu, G. Kioseoglou, and A. Petrou, Kinetic and transport modeling of the metallorganic chemical vapor deposition of InP from trimethylindium. J. Electrochem. Soc. 142, 2086-2094 (1995).

[41] A. Theodoropolou, R.A. Adomaitis, and E. Zafiriou, Model reduction for optimization of rapid thermal chemical vapor deposition systems, IEEE Trans. Semiconductor Manuf. 11, 85-98 (1998).

[42] S.C. Warnick and M.A. Dahleh, Feedback control of MOCVD growth of submicron compound semiconductor films, IEEE Trans. Control Systems Tech. 6, 62-71 (1998).

[43] J.J. Zhou, Y. Li, D. Pacheco, H.P. Lee, and X. Liu, Virtual control simulator for closed-loop epitaxial growth, J. Crystal Growth 175/176, 52-60 (1997). 